\title{
Ultralow loss polycrystalline alumina
}

\author{
Jonathan D. Breeze, ${ }^{\text {a) }}$ Xavi Aupi, and Neil McN. Alford ${ }^{\text {b) }}$ \\ Centre for Physical Electronics and Materials, South Bank University, London, SE1 OAA, United Kingdom
}

(Received 9 September 2002; accepted 1 November 2002)

\begin{abstract}
Polycrystalline alumina with extremely low microwave dielectric loss is reported with properties analogous to a theoretical ensemble of randomly oriented, single crystal sapphire grains. By avoiding deleterious impurities and by careful control of microstructure, we show that grain boundaries in aluminum oxide have only a limited influence on the dielectric loss. A method of measuring the electric permittivity and loss tangent of low-loss microwave ceramic dielectrics is reported. The electrical parameters such as relative permittivity and loss tangent are extracted using the radial mode matching technique. The measured values for ultralow loss polycrystalline aluminum oxide agree well with theoretical values modelled on an ensemble of randomly oriented anisotropic single crystal sapphire grains. () 2002 American Institute of Physics.
\end{abstract}

[DOI: $10.1063 / 1.1532553$ ]

Microwave dielectric ceramics with extremely low loss $\left(\tan \delta<10^{-5}\right)$ and relatively low permittivity $\left(\varepsilon_{r} \sim 10\right)$ such as sapphire $\left(\mathrm{Al}_{2} \mathrm{O}_{3}\right)$ and $\mathrm{MgO}$, have applications in microwave communication in the $10-50 \mathrm{GHz}$ frequency band. They also have application as resonators for ultrastable oscillators in clocks. Single crystals are used due to their low losses in the range of $10^{-5}$ at $10 \mathrm{GHz} .^{1}$ Polycrystalline ana$\operatorname{logs}$ of these materials usually have similar permittivity, but losses that are generally a factor of at least two higher. The measurement of low loss, low permittivity microwave dielectric ceramics using the $\mathrm{TE}_{01 \delta}$ is limited in accuracy by the electric filling factor and geometric factor of the conducting shield. By carefully choosing the dimensions of the conducting shield for optimum electric filling factor $P_{e}$ and geometric factor $G$, it is possible to measure the $\tan \delta$ with $10 \%$ accuracy for $\tan \delta>10^{-5} .^{2}$ The preferred method of measurement for $\tan \delta<10^{-6}$ utilizes high order whispering gallery modes, ${ }^{3,4}$ which have electric filling factors approaching unity and very high geometric factors. Unfortunately, the modal number density is usually very high in the frequency range in which whispering gallery modes exist, and careful choice of cavity dimensions is necessary to avoid spurious modes. ${ }^{5}$ This letter seeks to extend the usefulness of the $\mathrm{TE}_{01 \delta}$ mode measurement method by eliminating a great source of error: the support. The relative permittivity of the dielectric resonator can be calculated using the radial mode matching method. ${ }^{6}$ Instead of solving for frequency, as is usually done, one can instead solve for $\varepsilon_{r}$. After calculation of the electric filling factor and geometric factors, the $\tan \delta$ can be obtained from the expression

$$
\tan \delta=P_{e}^{-1}\left(Q_{0}^{-1}-R_{S} G^{-1}-P_{\text {spacer }} \tan \delta_{\text {spacer }}\right),
$$

where $P_{e}$ is the electric filling factor, $Q_{0}$ is the unloaded quality factor, $R_{S}$ is the surface resistivity, and $G$ is the geometric factor.

An alumina dielectric resonator was constructed as shown in Fig. 1 using the $\mathrm{TiO}_{2}$-doped $\mathrm{Al}_{2} \mathrm{O}_{3}$ developed in

\footnotetext{
${ }^{a)}$ Electronic mail: breezejd@sbu.ac.uk

${ }^{b)}$ Electronic mail: alfordn@sbu.ac.uk
}

the laboratory. ${ }^{7}$ This material is high purity and fine grained, both of which are necessary in order to achieve very low loss. ${ }^{8,9}$ The dimensions of the conducting shield are diameter $36.00 \mathrm{~mm}$, height $23.48 \mathrm{~mm}$. The alumina puck has diameter $10.69 \mathrm{~mm}$ and height $4.34 \mathrm{~mm}$. The alumina spacer has diameter $4.13 \mathrm{~mm}$ and height $6.77 \mathrm{~mm}$.

The resonant frequency of the quasi- $\mathrm{TE}_{011}\left(\mathrm{TE}_{01 \delta}\right)$ mode was measured at room temperature $(300 \mathrm{~K})$ and found to be $8.95557 \mathrm{GHz}$. The measurement was performed in transmission $\left(S_{21}\right)$ using an Agilent network analyzer (HP8722). Input and output coupling to the resonator was achieved using coaxial transmission lines with small loops formed by soldering the central conductors to the outer shield of the coaxial cable. The coupling was $-40.5 \mathrm{~dB}$, measured from the insertion loss. The loaded quality factor $Q_{L}$ of the mode was measured and found to be 62500 . For equal input and output couplings, the unloaded quality factor $Q_{0}$ can be calculated as

$$
Q_{0}=\frac{Q_{L}}{1-\left|S_{21}\right|} .
$$

In this case, $Q_{0}$ was found to be 63090 . The resistivity of the oxygen-free copper shield was found by measuring $Q_{0}$ for the $\mathrm{TE}_{111}$ and $\mathrm{TE}_{011}$ modes of the empty cavity (without dielectric). This was done in order to confirm the frequency dependence. These measurements were performed as a function of temperature using a Gifford-McMahon closed-cycle cooler from 20 to $320 \mathrm{~K}$. At room temperature and 8.95556 $\mathrm{GHz}$, the resistivity was calculated to be $2.24 \times 10^{-8} \Omega \mathrm{m}$. The surface resistance $R_{s}$ in this case was $28.15 \mathrm{~m} \Omega$.

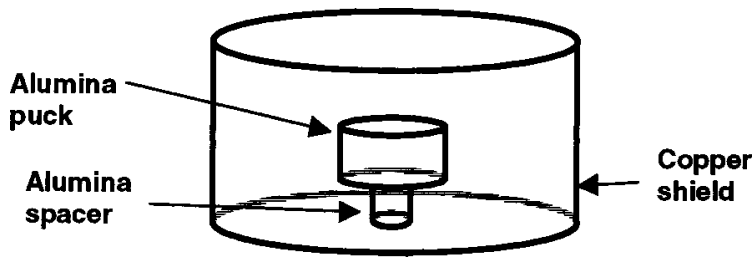

FIG. 1. Structure of alumina dielectric resonator. 
TABLE I. Comparison of filling factors and geometric factors using different modelling methods.

\begin{tabular}{cccc}
\hline \hline & $\begin{array}{c}\text { Mode } \\
\text { Matching }\end{array}$ & MAFIA & $\begin{array}{c}\text { Finite difference } \\
\text { time domain }\end{array}$ \\
\hline Electric filling factor $P_{e}$ & 0.777 & 0.770 & 0.775 \\
Geometric factor $G$ & 2812 & 2669 & 2825 \\
\hline \hline
\end{tabular}

The $\varepsilon_{r}$ of the alumina was calculated using the radial mode matching method. This entails solving a linear system of equations for a prescribed number of eigenmodes $N$. For this problem, $N=9$ modes were found to be sufficient for convergence of the solution. The value of $\varepsilon_{r}$ in this case for polycrystalline alumina is 10.15 . The $\tan \delta$ can be calculated using the expression

$$
\tan \delta=P_{e}^{-1}\left(Q_{0}^{-1}-R_{s} G^{-1}\right) .
$$

After measuring the unloaded quality factor $Q_{0}$ and surface resistivity $R_{s}$, it is necessary to calculate the electric filling factor and the geometric factors, using

$$
P_{e}=\frac{\iiint_{V_{d}} \varepsilon_{r} \mathbf{E} \cdot \mathbf{E}^{*} d V}{\iiint_{V} \varepsilon_{r}(V) \mathbf{E} \cdot \mathbf{E}^{*} d V},
$$

and

$$
G=\frac{\omega \iiint_{V} \mu_{0} \mathbf{H} \cdot \mathbf{H}^{*} d V}{\oiint_{S} \mathbf{H}_{t} \cdot \mathbf{H}_{t}^{*} d S} .
$$

These quantities can be evaluated once the field coefficients of the series expansion are known. Surface and volume integration of the fields allows one to calculate $P_{e}$ and $G$. Alternatively, the incremental frequency rule can be used. ${ }^{9,10}$ The values of $P_{e}$ and $G$ were calculated to be 0.777 and 2812, respectively. These values were compared with results from a two-dimensional finite difference time domain $\operatorname{code}^{11}$ and MAFIA. ${ }^{12}$ These results are given in Table I. From $G$ and $R_{s}$ we derive a conductor quality factor $Q_{c}$ of 99893 . This results in a dielectric quality factor $Q_{d}$ of 171241 . We can now calculate the loss tangent,

$$
\tan \delta=\frac{1}{P_{e} Q_{d}} .
$$

The result is $\tan \delta=7.516 \times 10^{-6}$ at a frequency of 8.95556 $\mathrm{GHz}$. Assuming a linear frequency dependence for the loss in the frequency range from 8 to $10 \mathrm{GHz}$, allows the loss tangent to be normalized to $10 \mathrm{GHz}\left(8.393 \times 10^{-6}\right)$. Hence a $Q f$ product of 1191580 . The properties are summarized in Table II.

For comparison, Krupka et $a l^{3}{ }^{3}$ reported a relative permittivity tangential to the $c$-axis, $\varepsilon_{t}$, of 9.400 , and parallel to the axis, $\varepsilon_{z}$, of 11.587 for single crystal sapphire. Also reported ${ }^{4}$ were values for the $\tan \delta$ for the transverse plane,

TABLE II. Dielectric properties of polycrystalline $\mathrm{TiO}_{2}$-doped $\mathrm{Al}_{2} \mathrm{O}_{3}$.

\begin{tabular}{ll}
\hline \hline Relative permittivity $\varepsilon_{r}$ & 10.15 \\
$\tan \delta$ measured at $8.95557 \mathrm{GHz}$ & $7.516 \times 10^{-6}$ \\
$\tan \delta$ normalized to $10 \mathrm{GHz}$ & $8.393 \times 10^{-6}$
\end{tabular}

$\tan \delta_{t}$ of $9.09 \times 10^{-6}$ at $9.02985 \mathrm{GHz}$, and $4.76 \times 10^{-6}$ at $8.85522 \mathrm{GHz}$ for the axial component $\tan \delta_{z}$. By assuming that the frequency dependence of the loss tangent is linear, we can normalize all these values to $10 \mathrm{GHz}$ for comparison. The normalized $\tan \delta$ for the results reported by Krupka et al. ${ }^{3}$ are transverse $\tan \delta=1.01 \times 10^{-5}$ and axial $\tan \delta$ $=5.38 \times 10^{-6}$. An effective loss tangent can be calculated in the same way as the permittivity; using the equation for effective permittivity, the result is $\tan \delta=8.33 \times 10^{-6}$. It is interesting to note that the $\tan \delta$ value for the polycrystalline alumina measured here and normalized to $10 \mathrm{GHz}$ is $\tan \delta$ $=8.39 \times 10^{-6}$.

The problem of the effective permittivity of an ensemble of randomly oriented uniaxial spherical crystallites has been investigated by numerous authors. The following equation was derived by Herring, ${ }^{13}$

$$
\varepsilon^{\prime}=\frac{1}{3} \varepsilon_{z}+\frac{2}{3} \varepsilon_{t}-\frac{2}{9} \frac{\left(\varepsilon_{z}-\varepsilon_{t}\right)^{2}}{\varepsilon_{z}+2 \varepsilon_{t}},
$$

where the strong isotropy condition is assumed. A generalized form for the biaxial case was reported by Stroud: ${ }^{14}$

$$
\varepsilon^{\prime}=\bar{\varepsilon}-\frac{1}{9 \bar{\varepsilon}} \sum_{i=1}^{N}\left(\varepsilon_{i}-\bar{\varepsilon}\right)^{2},
$$

which is identical in form to that reported by Landau et al. ${ }^{15}$ for a dielectric mixture.

Using the sapphire measurements of Krupka et al. to calculate the properties of polycrystalline alumina yields a relative permittivity $\varepsilon_{r}$ of 10.094 and loss tangent $\tan \delta=8.33$ $\times 10^{-6}$. We show that high purity, fine grained polycrystalline alumina possesses very low dielectric loss. A $\tan \delta$ of $8.33 \times 10^{-6}$ was measured at $10 \mathrm{GHz}$ and at $300 \mathrm{~K}$. Using literature values for the $\tan \delta$ of sapphire, parallel and normal to the $c$-axis, we modelled the dielectric properties. We obtained very good agreement between the results of the model and the experimental observations.

This work was supported by the Engineering and Physical Sciences Research Council and by the European Union Framework V program. We thank L. Hao of the National Physical Laboratory for providing modelled data using the MAFIA code.

${ }^{1}$ M. E. Tobar, J. Krupka, E. N. Ivanov, and R. A. Woode, J. Appl. Phys. 83, 1604 (1998)

${ }^{2}$ J. Krupka, Special Session for Microwave Materials, Asia Pacific Microwave Conference, Taipei, 2001, pp. 1-47 (unpublished abstracts).

${ }^{3}$ J. Krupka, K. Derzakowski, M. Tobar, J. Hartnett, and R. G. Geyer, Meas. Sci. Technol. 10, 387 (1999).

${ }^{4}$ J. Krupka, K. Derzakowski, A. Abramowicz, M. E. Tobar, and R. G. Geyer, IEEE Trans. Microwave Theory Tech. 47, (1999).

${ }^{5}$ C. Wang and K. A. Zaki, IEEE Trans. Microwave Theory Tech. 48, 60 (2000).

${ }^{6}$ D. Kajfez and P. Guillon, Dielectric Resonators (Noble, Atlanta, 1998).

${ }^{7}$ Neil McN. Alford and Stuart J. Penn, J. Appl. Phys. 80, 5895 (1996).

${ }^{8}$ S. J. Penn, N. McN. Alford, A. Templeton, X. Wang, M. Xu, M. Reece, and Kevin Schrapel, J. Am. Ceram. Soc. 80, 1885 (1997).

${ }^{9}$ D. Kajfez, IEEE Trans. Microwave Theory Tech. 32, 941 (1984).

${ }^{10}$ Y. Kobayashi, T. Aoki, and Y. Kabe, IEEE Trans. Microwave Theory Tech. 33, 1361 (1985).

${ }^{11}$ A. Navarro, M. J. Nuñez, and E. Martin, IEEE Trans. Microwave Theory Tech. 39, 14 (1991).

${ }^{12}$ M. Bartsch, M. Dehler, M. Dohlus, F. Ebeling, P. Hahne, R. Klatt, F. Downloaded 18 Dec 2002 to 192.58.150.40. Redistribution subject to AIP license or copyright, see http://ojps.aip.org/aplo/aplcr.jsp 
Krawczyk, M. Marx, P. Schütt, B. Steffen, T. Pröpper, D. Schmitt, T. Weiland, S. Wipf, H. Wolter, and M. Zhang, Comput. Phys. Commun. 72, 22 (1992).

${ }^{13}$ C. Herring, J. Appl. Phys. 31, 1939 (1960).
${ }^{14}$ D. Stroud and A. Kazaryan, Phys. Rev. B 53, 7076 (1996).

${ }^{15}$ L. D. Landau, E. M. Lifshitz, and L. P. Piaevskii, Electrodynamics of Continuous Media, Vol. 8, edited by E. M. Lifshitz (ButterworthHeinmann, Washington, D.C., 1995). 